\title{
LeVANTAMENTo ETNOBOtÂNiCo do JATOBÁ (HyMENAEA COURbariL L., Fabaceae) no norte do estado de Mato Grosso, Brasil
}

\author{
Poliana Vicente Tiago ${ }^{1}$, Ana Aparecida Bandini Rossi², Eliane Cristina Moreno de Pedri ${ }^{3 *}$, José Martins \\ Fernandes $^{2}$, Auana Vicente Tiago ${ }^{3}$, Joameson Antunes Lima ${ }^{4}$
}

\author{
1 Programa de Pós-Graduação em Biodiversidade e Agroecossistemas Amazônicos, UNEMAT/Alta Floresta, MT. \\ 2 Docente da Universidade do Estado de Mato Grosso, UNEMAT/ Alta Floresta, MT. \\ 3 Programa de Pós-Graduação em Biodiversidade e Biotecnologia, UNEMAT/Alta Floresta, MT. \\ 4 Programa de Pós-Graduação em Genética e Melhoramento de Plantas, UNEMAT/Alta Floresta, MT. \\ *e-mail do autor para correspondência: elicmbio@gmail.com
}

Recebido em 22 de janeiro de 2019. Aceito em 21 de julho de 2019. Publicado em 31 de julho de 2019.

\begin{abstract}
Resumo - O jatobá (Hymenaea courbaril L.), pertencente à família Fabaceae, é uma planta arbórea que possui potencial madeireiro, alimentar e medicinal. Este estudo teve por objetivo realizar um levantamento etnobotânico do jatobá (Hymenaea courbaril) na região norte do Estado de Mato Grosso, Brasil. Dados socioeconômicos e etnobotânicos foram obtidos a partir de perguntas fechadas e abertas com os entrevistados. Os resultados foram avaliados quali e quantitativamente. Os entrevistados, em sua maioria, nasceram no Sul do Brasil, mas residem há mais de 20 anos no norte de Mato Grosso, com predominância de agricultores familiares. Os entrevistados relataram conhecer a espécie, principalmente, pelas características das folhas, casca e fruto. A espécie é utilizada nas categorias alimentar, artesanato, ecológica, madeireira, medicinal e tecnológica, totalizando 42 tipos de usos, sendo que destes, $83 \%$ foram incluídos na categoria medicinal. As maiores concordâncias de uso entre os entrevistados foram para o tratamento de gripe $(\mathrm{CUPc}=68)$, anemia $(\mathrm{CUPc}=56)$ e farinha do fruto para alimento $(\mathrm{CUPc}=48)$. Os resultados indicam que $H$. courbaril é uma espécie de uso múltiplo, sendo utilizado, principalmente, como medicinal e que os entrevistados possuem amplo conhecimento sobre a espécie nativa da Amazônia Brasileira.
\end{abstract}

Palavras-chave: Amazônia, conservação, uso múltiplo.

Ethnobotanical survey of Jatoba (Hymenaea courbaril L., Fabaceae) in northern Mato Grosso state, Brazil

Aвstract - The jatoba (Hymenaea courbaril L.) belongs to the Fabaceae family and is a woody plant that has potential for timber industry, food and medicine. The objective of this study was to conduct a survey and ethnobotanical characterization of jatoba (Hymenaea courbaril) in the northern region of the state of Mato Grosso, Brazil. Socioeconomic and ethnobotanical data were obtained from objective and dissertative questions with the interviewees. The results were evaluated qualitatively and quantitatively. Most of the interviewees were born in the South of Brazil, but have lived for more than 20 years in the north of Mato Grosso, with predominance of family farmers. The interviewees reported knowing the species, mainly, by the characteristics of leaves, bark and fruit. The species is used in the categories of food, handicrafts, ecological, timber industry, medicinal and technological, totaling 42 types of uses, of which $83 \%$ were included in the medicinal category. The highest agreement of use among the interviewees was for the treatment of influenza $(\mathrm{CUPc}=68)$, anemia $(\mathrm{CUPc}=56)$ and fruit flour for food $(\mathrm{CUPc}=48)$. The results indicate that $H$. courbaril is a species of multiple uses, being used, mainly, as medicinal. The interviewees have extensive knowledge about this native species of the Brazilian Amazon.

KEywords: Amazon, conservation, multiple use. 
Levantamiento etnobotánico del jatobá (Hymenaea courbaril L., Fabaceae) en el norte del estado de Mato Grosso, BrasIL

RESUMEN - El jatobá (Hymenaea courbaril L.), perteneciente a la familia Fabaceae, es una planta arbórea que posee potencial maderero, alimentario y medicinal. Este estudio tuvo por objetivo realizar un levantamiento y caracterización etnobotánica del jatobá (Hymenaea courbaril) en la región norte del Estado de Mato Grosso, Brasil. Los datos socioeconómicos y etnobotánicos se obtuvieron a partir de preguntas cerradas y abiertas con los entrevistados. Los resultados se evaluaron cuantitativamente y cuantitativamente. Los entrevistados, en su mayoría, nacieron en el sur de Brasil, pero residen desde hace más de 20 años en el norte de Mato Grosso, con predominio de agricultores familiares. Los entrevistados relataron conocer la especie, principalmente, por las características de las hojas, cáscara y fruto. La especie se utiliza en las categorías alimentaria, artesanal, ecológica, maderera, medicinal y tecnológica, totalizando 42 tipos de usos, siendo que de éstos, 83\% fueron incluidos en la categoría medicinal. Las mayores concordancias de uso entre los entrevistados fueron para el tratamiento de gripe $($ CUPc $=68)$, anemia $(C U P c=56)$ y harina del fruto para alimento $(C U P c=48)$. Los resultados indican que $H$. courbaril es una especie de uso múltiple, siendo utilizado, principalmente, como medicinal y que los entrevistados poseen amplio conocimiento sobre la especie nativa de la Amazonia Brasileña.

Palabras clave: Amazonia, conservación, uso múltiple.

\section{INTRODUÇÃO}

Fabaceae com 766 gêneros e cerca de 19.580 espécies é considerada a terceira maior família de angiospermas, apresentando distribuição cosmopolita, reconhecida pela presença de folhas compostas, alternas e com pulvinos, uma pétala adaxial diferenciada, ovário unicarpelar e fruto do tipo legume (Lewis et al. 2005; APW 2019). No Brasil está amplamente distribuída em todos os domínios fitogeográficos, constituída por 222 gêneros e 2.846 espécies (BFG 2018).

Entre as espécies nativas no Brasil, encontra-se Hymenaea courbaril Linnaeus, uma espécie arbórea com registro de ocorrência nas regiões norte, nordeste, centro-oeste, sudeste e sul do país, com grande utilidade e potencial econômico (BFG 2018; Lima e Pinto 2015).

Os jatobazeiros apresentam troncos retos e cilíndricos, de súber liso e de coloração cinza; no Brasil, a floração ocorre durante os meses de dezembro a fevereiro e a frutificação entre os meses de agosto e setembro; suas flores são monóclinas, diclamídeas e pentâmeras, com cálice dialissépalo e corola dialipétala, com 10 estames e um pistilo; o fruto é do tipo legume nucóide com arilo farináceo de sabor adocicado, com elevado teor de fibra alimentar e grande quantidade de cálcio (Ca) e magnésio $(\mathrm{Mg})$, sendo utilizado na alimentação humana e animal (Barroso 1991; Batista et al. 2011).

O jatobá apresenta potencial econômico, pois fornece madeira resistente e de boa qualidade sendo empregada na construção civil e em carpintaria; a resina (jutaicica) do jatobá é utilizada na fabricação de verniz (Lisboa et al. 1991; Alvino et al. 2005) e na medicina popular no tratamento da bronquite, asma, deficiência pulmonar e laringite (Salman et al. 2008).

Na Amazônia brasileira, o jatobá é comumente utilizado como fitoterápico tradicional (Guarim Neto 1997), contudo o principal uso econômico é a madeira que está entre as mais valiosas e exploradas (Lacerda et al. 2008). A espécie vem sofrendo efeitos negativos devido a expansão das fronteiras agrícolas e a exploração desenfreada da madeira (Castellenn 2005; Toledo 2005). No estado do Mato Grosso o reflorestamento induzido é uma alternativa viável e adotada para a recuperação de áreas degradadas e uma das espécies indicadas para este fim é o jatobá (Silva 2017).

A interação entre a sociedade e as plantas no seu ambiente é estudada pela etnobotânica, ciência que realiza o registro dos recursos vegetais e as formas de manejo empregado pela comunidade (Silva et al. 2015). O etnoconhecimento das espécies vegetais associado ao uso e manejo consciente é uma alternativa de subsistência 
e manutenção da diversidade cultural e vegetal (Pereira et al. 2012). E como destaca Pasa et al. (2005) é, em muitos casos, o único recurso disponível que a população rural de países em desenvolvimento tem ao seu alcance.

Estudos etnobotânicos têm sido desenvolvidos em distintas regiões brasileiras com o gênero Hymenaea (Albuquerque e Andrade 2002; Pasa et al. 2005; Cunha e Bortolotto 2011; Vásquez et al. 2014), porém, apesar da espécie ter ocorrência natural na região e um amplo histórico de utilização, ainda são poucos os trabalhos encontrados, dentro dessa temática, para o jatobá. Portanto, estudos etnobotânicos, por meio de suas investigações, promoverá a divulgação das potencialidades da espécie, já que hábitos tradicionais de cultivo estão sendo perdidos ao longo das gerações devido ao processo de modernização, levando a facilidade de obtenção de produtos no comércio (Gandolfo e Hanazaki 2011; Fagundes et al. 2017).

Diante do exposto, o objetivo do estudo foi realizar um levantamento etnobotânico do jatobá (Hymenaea courbaril L.) na região norte do estado de Mato Grosso, Brasil.

\section{Material e Métodos}

A pesquisa foi realizada nos municípios de Alta Floresta, Carlinda e Nova Bandeirantes, ambos localizados no norte do estado de Mato Grosso (Figura 1A). O município de Alta Floresta, distante a 797 km da capital, Cuiabá, abrange uma área de $8.953,191 \mathrm{~km}^{2}$ (Figura 1B). Carlinda dista a $757 \mathrm{~km}$ da capital, com área de $2.416,144 \mathrm{~km}^{2}$ (Figura 1C). Nova Bandeirantes, distante a $998 \mathrm{~km}$ de Cuiabá, apresenta uma área de 9.593,268 km² (Figura 1D). Os municípios apresentam população estimada, em 2018, de 51.615 (13\% residente na zona rural), 10.413 (58\% na zona rural) e 14.909 habitantes ( $65 \%$ na zona rural), respectivamente, tendo como principais atividades a criação de bovinos e suínos, cultivo de café, guaraná, milho, feijão, arroz e, mais recentemente, a soja, várias espécies de peixes para comercialização ou subsistência, produção de mel de abelhas nativas (abelhas sem ferrão) e introduzidas (Apis mellifera L.), extração vegetal de espécies nativas para produção de madeira e carvão e o ecoturismo como atividade turística (IBGE 2018). O clima dos municípios é do tipo Am (tropical e subtropical), com estação chuvosa e seca, temperatura média anual de $26{ }^{\circ} \mathrm{C}$ e precipitação variando entre 2800 e $3100 \mathrm{~mm}$ (Alvares et al. 2013).

Figura 1. Localização geográfica dos municípios de Alta Floresta (B), Carlinda (C) e Nova Bandeirantes (D), ambos localizados no norte do estado de Mato Grosso.

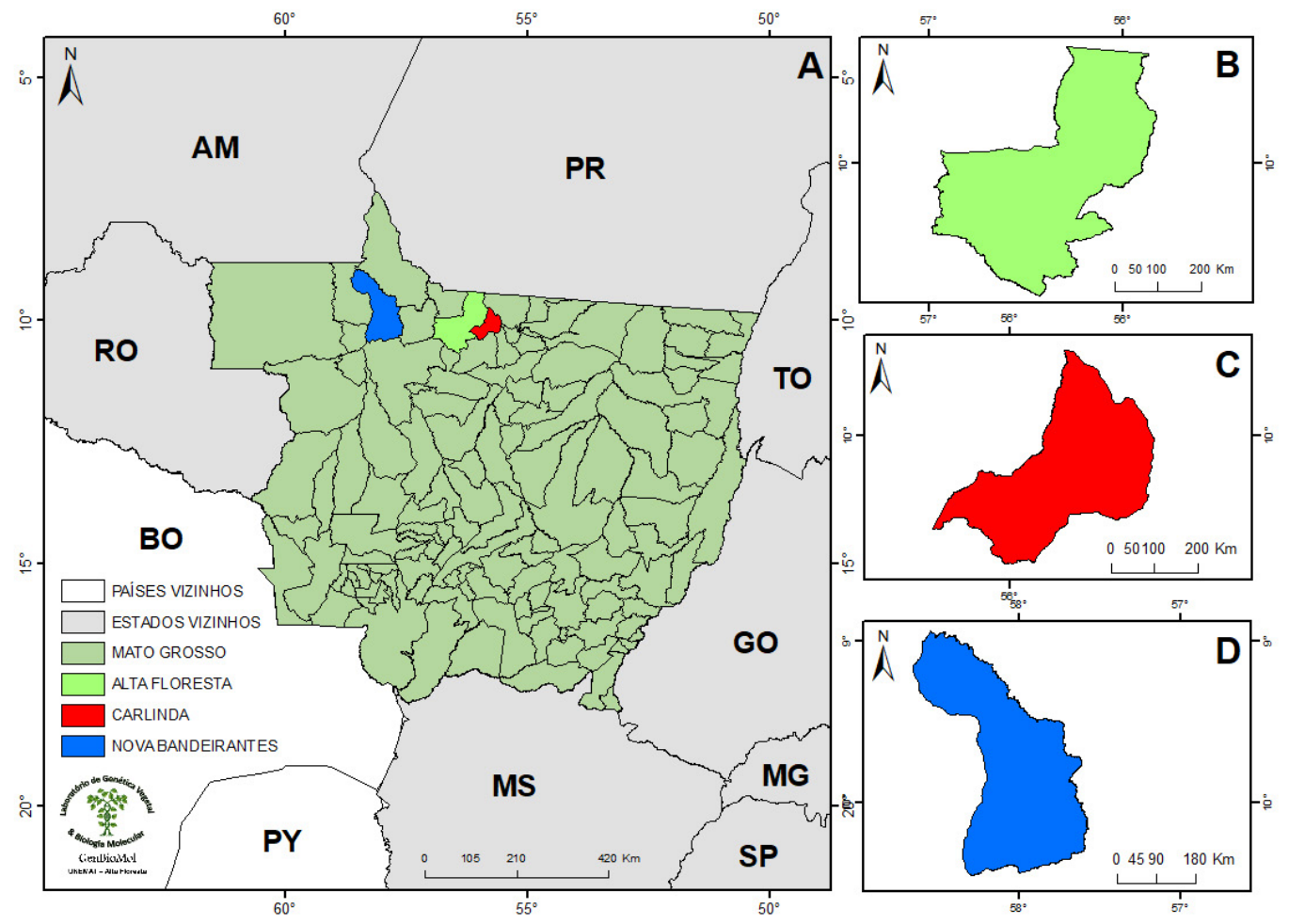


Primeiramente foi realizada uma visita na feira livre do município de Alta Floresta, MT, local de comercialização de produtos agropecuários e plantas medicinais, dentre elas o jatobá, e a partir de então, pelo método de amostragem bola de neve (Albuquerque e Lucena 2004), foram selecionadas as pessoas que conhecem e utilizam a espécie, chegando assim aos demais municípios amostrados. Segundo Albuquerque e Lucena (2004), o método "bola de neve" é uma forma de seleção de informantes não-probalística, onde a partir do contato inicial com a comunidade, um primeiro especialista é reconhecido, que passa a indicar outro especialista e assim, sucessivamente, até envolver todos os especialistas da comunidade.

O estudo ocorreu entre dezembro de 2014 e janeiro de 2016 a partir de entrevistas, com perguntas fechadas e abertas, com 26 entrevistados (um por família) sendo que, destes, dezessete (65\%) foram de Alta Floresta, cinco $(19 \%)$ de Carlinda e quatro (15\%) de Nova Bandeirantes.

O projeto de pesquisa foi submetido à apreciação do Comitê de Ética em Pesquisa da Universidade do Estado de Mato Grosso (UNEMAT) sob o número 52641915.8.0000.5166, parecer consubstanciado 1.507.091 aprovado em 19 de abril de 2016.

A primeira etapa da pesquisa constituiu em um levantamento, junto aos conhecedores do jatobá, quanto ao conhecimento e uso da espécie. No primeiro contato, foi apresentado o projeto de pesquisa e o Termo de Consentimento Livre e Esclarecido (TCLE), onde os entrevistados eram livres para participar ou não da pesquisa.

Após a assinatura do termo, os entrevistados responderam a um questionário onde, além de informações pessoais, foram questionados sobre o conhecimento e uso do jatobá. As respostas dos entrevistados foram anotadas e gravadas com o consentimento dos mesmos a fim de guardar registro fidedigno das falas dos entrevistados.

O guia de entrevista foi dividido em duas partes: informações pessoais e socioeconômicas (10 questões) e conhecimento e uso do jatobá (17 questões). Os resultados foram analisados de maneira quali e quantitativamente aplicando-se os Índices de Concordância de Uso adaptado por Amorozo e Gély (1988), utilizando Microsoft Excel. Estes índices são utilizados para avaliar a importância relativa da espécie entre os entrevistados com três ou mais citações, bem como sua concordância de uso. Os cálculos foram realizados de acordo com a equação 1:

$$
\mathrm{CUP}=\mathrm{NECUP} / \mathrm{NECC} \times 100
$$

onde,

CUP $=\%$ de concordância para cada utilização

NECUP $=\mathrm{n}^{\circ}$ de entrevistados que citam determinada utilização para o gengibre

$\mathrm{NECC}=\mathrm{n}^{\circ}$ de entrevistados que citam a categoria de utilização

Posteriormente, foram calculados os fatores de correção para cada uma das categorias (equação 2) e o valor de importância de cada forma de utilização relativa à categoria mais citada pelos entrevistados (equação 3).

$$
\begin{gathered}
\mathrm{FC}=\mathrm{NECC} / \mathrm{NECCM} \\
\mathrm{CUPc}=\mathrm{CUP} \times \mathrm{FC}
\end{gathered}
$$

sendo,

$\mathrm{FC}=$ fato de correção da categoria

NECC $=n^{\circ}$ de entrevistados que citam a categoria de utilização

$\mathrm{NECCM}=\mathrm{n}^{\circ}$ de entrevistados que citaram a categoria mais utilizada

CUPc $=$ valor de importância relativa à forma de utilização mais citada pelos entrevistados 
Durante o estudo etnobotânico, amostras férteis de jatobá foram coletadas seguindo técnicas usuais (Fidalgo e Bononi 1989) e depositadas no Herbário da Amazônia Meridional (HERBAM) da Universidade do Estado de Mato Grosso Carlos Alberto Reyes Maldonado (UNEMAT), Campus de Alta Floresta, Mato Grosso. Foram identificadas com base na revisão taxonômica de Lee e Langenheim (1975) e a partir de comparação com exsicatas depositadas na coleção do HERBAM.

\section{Resultados e Discussão}

\section{Aspectos socioeconômicos dos entrevistados}

Foram entrevistadas 26 pessoas, sendo 13 mulheres e 13 homens. A idade dos entrevistados nesse estudo variou entre 25 e 82 anos, sendo 1 jovem (<29 anos), 13 pessoas entre 29 e 58 anos, 11 entre 60 e 77 anos e uma pessoa acima de 80 anos, demonstrando o predomínio dos mais velhos como maiores detentores do conhecimento sobre plantas, correlação também constatada por outros autores (Campos et al. 2015; Messias et al. 2015; Prado et al. 2019).

Quanto ao estado civil dos entrevistados dezenove são casados (73\%), quatro são viúvos (a) (15\%), dois solteiros (a) (8\%) e um divorciado (a) (4\%). Sessenta e cinco por cento dos entrevistados residem na zona urbana $(\mathrm{n}=17)$ e $35 \%$ na zona rural $(\mathrm{n}=9)$.

Quanto a escolaridade, três $(12 \%)$ dos entrevistados se declararam analfabetos, dois (8\%) foram apenas alfabetizados (fora do ambiente escolar), nove (35\%) possuem o Ensino Fundamental Incompleto, dois (8\%) cursaram o Ensino Fundamental Completo, dois ( $8 \%$ ) possuem Ensino Médio Incompleto e três (12\%) Ensino Médio Completo. Apenas cinco (19\%) entrevistados possuem nível superior.

Os entrevistados são naturais das regiões sul $(46 \% ; n=12)$, norte $(27 \% ; n=7)$, sudeste $(23 \% ; n=6)$ e centrooeste $(04 \% ; n=1)$ do país, sendo, na sua maioria, do Sul do Brasil. Este fato está relacionado ao processo de colonização da região Amazônica, que atraídos pelas políticas públicas de ocupação, se deslocaram com o objetivo de adquirir terras em busca de melhor qualidade de vida (Seluchinesk 2008; Perin 2015). Com o passar do tempo à região recebeu colonos das mais distintas regiões do Brasil, colonos estes que trouxeram consigo diversos conhecimentos populares e implantaram na região o cultivo de culturas perenes como café, cacau e guaraná, que posteriormente foram convertidas em pastagens (Roboredo et al. 2017).

Os entrevistados citaram 14 profissões distintas, destacando-se a agricultura familiar com 31\% ( $\mathrm{n}=10$ ). Dentre os entrevistados, 20 deles residem entre 20 e 39 anos na região e apenas seis entre um (01) e 19 anos, evidenciando que os entrevistados tiveram tempo de interagir com os recursos da vegetação local e diferentes povos na região.

Os entrevistados declararam obter os recursos do jatobá predominantemente nas próprias propriedades $(62 \% ; n=16)$, de vizinhos $(19 \% ; n=5)$, amigos $(4 \% ; n=1)$ ou até mesmo em comércio local $(15 \% ; n=4)$.

\section{Conhecimento local sobre a espécie Hymenaea courbaril L. (jatobá)}

Todos os entrevistados conhecem popularmente a espécie $H$. courbaril como jatobá e citaram características morfológicas que ajudam no reconhecimento da espécie, como as folhas $(32 \% ; n=17)$, casca $(23 \% ; n=12)$, fruto $(19 \% ; n=10)$, porte arbóreo $(19 \% ; n=10)$, flor $(2 \% ; n=1)$, madeira $(2 \% ; n=1)$, resina $(2 \% ; n=1)$ e semente $(2 \%$; $\mathrm{n}=1)$. 
Os entrevistados associam a morfologia das folhas com a planta popularmente conhecida como pata de vaca (Baubinia spp.) pelo fato de apresentar, geralmente, as folhas bipartidas. Ainda quanto à folha, deram importância para o brilho, além de lisas e largas. Outra característica que ajuda no reconhecimento da espécie, segundo os entrevistados, é quanto à estrutura externa da casca, parecida com a casca da castanha do Brasil (Bertholletia excelsa). Outra parte da planta mencionada foram os frutos (10 citações) que são grandes, de cor marrom e de fácil visualização quando estão nas copas das árvores.

\begin{abstract}
Entrevistado 01: "Pela folha, pela casca quando tem o fruto também de longe a pessoa conhece ele, quando tá novo você conhece pela folha que a folha dele é tipo uma pata de vaca folha dele é aberta né com duas e quando ele tá alto reconhece pelo troco dele pelo caule que a casca dele é diferente né a casca dele é lisa e é bem branca, não tem outra árvore que parece com ele não".
\end{abstract}

As populações humanas estabelecem seus critérios de identificação e designação de recursos naturais, formando seus próprios sistemas de classificação da natureza, que podem ser investigados através de estudos da biologia popular (Ramires et al. 2012).

\title{
Categorias e tipos de uso do Jatobá
}

O jatobá é utilizado pelos agricultores dos três municípios estudados em 42 formas de uso, havendo 120 citações, classificadas em seis categorias (alimentícia, artesanato, ecológico, madeireiro, medicinal e tecnológico), sendo a categoria medicinal a mais citada entre os entrevistados (79\%; N=95) (Tabela 1). O uso do chá para tratar a gripe foi o mais expressivo pelos entrevistados $(\mathrm{N}=17)$, seguido do uso da farinha do fruto $(\mathrm{N}=12)$. Porém, vale ressaltar que a espécie apresenta uso múltiplo, destacando-se também o tratamento de anemia $(\mathrm{N}=14)$, infecção pulmonar $(\mathrm{N}=8)$, madeira para construção $(\mathrm{N}=7)$, bronquite $(\mathrm{N}=4)$, infecção geral $(\mathrm{n}=4)$ e reumatismo $(\mathrm{N}=4)$. A categoria de uso medicinal também foi mais representativa nos estudos etnobotânicos realizados por Cunha e Bortolotto (2011), Andrade et al. (2013), Ferreira et al. (2014), Moraes Rego et al. (2016) e Silva e Lamarca (2018) com distintas espécies medicinais.

Tabela 1. Categorias e tipos de uso do jatobá (Hymenaea courbaril) na região norte do estado de Mato Grosso. $\mathrm{F}=$ folha, $\mathrm{C}=$ casca, $\mathrm{Cl}=$ caule, $\mathrm{R}=$ raiz, $\mathrm{S}=$ semente $\mathrm{Ft}=$ fruto, $\mathrm{Fl}=$ flor.

\begin{tabular}{ccccc}
\hline Categoria & Tipos de uso & Citações & Parte usada & Preparo \\
\hline \multirow{2}{*}{ Alimentar } & Farinha do fruto & 12 & $\mathrm{Ft}$ & In natura \\
& Bebida & 1 & $\mathrm{C}$ & Curtida \\
\hline \multirow{2}{*}{ Artesanato } & Colar & 1 & $\mathrm{~S}$ & - \\
& Boneca & 1 & $\mathrm{Ft}$ & - \\
\hline Ecológica & Reflorestamento & 1 & $\mathrm{~S}$ & - \\
\hline Madeireira & Madeira construção & 7 & $\mathrm{Cl}$ & - \\
\hline
\end{tabular}




\begin{tabular}{|c|c|c|c|c|}
\hline Categoria & Tipos de uso & Citações & Parte usada & Preparo \\
\hline \multirow{35}{*}{ Medicinal } & Gripe & 17 & $\mathrm{C}$ & Infusão/Fervimento \\
\hline & Anemia & 14 & $\mathrm{C}$ & Fervimento \\
\hline & Infecção pulmonar & 8 & $\mathrm{C} / \mathrm{Ft}$ & Infusão/Fervimento \\
\hline & Bronquite & 4 & $\mathrm{C}$ & Fervimento \\
\hline & Infecção geral & 4 & $\mathrm{~F} / \mathrm{C}$ & Infusão \\
\hline & Reumatismo & 4 & $\mathrm{C}$ & $\mathrm{F} / \mathrm{S} / \mathrm{C}$ \\
\hline & Fortalecer o pulmão & 3 & $\mathrm{~F} / \mathrm{C}$ & Infusão \\
\hline & Imunidade baixa & 3 & $\mathrm{C} / \mathrm{Ft} / \mathrm{F}$ & Infusão/Fervimento \\
\hline & Infecção urinária & 3 & $\mathrm{C} / \mathrm{F} / \mathrm{Ft} / \mathrm{Fl} / \mathrm{S}$ & Infusão \\
\hline & Próstata & 3 & $\mathrm{C} / \mathrm{F}$ & Infusão/Fervimento \\
\hline & Vermífugo & 3 & $\mathrm{C}$ & Infusão/Fervimento \\
\hline & Cicatrizante & 2 & $\mathrm{C}$ & Fervimento \\
\hline & Emagrecedor & 2 & $\mathrm{~F} / \mathrm{C}$ & Infusão/ Fervimento \\
\hline & Infecção do intestino & 2 & $\mathrm{C}$ & Infusão \\
\hline & Pneumonia & 2 & $\mathrm{~F} / \mathrm{C}$ & Infusão \\
\hline & Útero & 2 & $\mathrm{C} / \mathrm{F} / \mathrm{Ft} / \mathrm{Fl} / \mathrm{S}$ & Infusão \\
\hline & Asma & 1 & $\mathrm{C}$ & Infusão \\
\hline & Baço & 1 & $\mathrm{C} / \mathrm{Ft}$ & Fervimento/In natura \\
\hline & Cistite & 1 & $\mathrm{C}$ & Infusão \\
\hline & Coração & 1 & $\mathrm{C}$ & Infusão/Fervimento \\
\hline & Depurativo do sangue & 1 & $\mathrm{C} / \mathrm{F}$ & Infusão/Fervimento \\
\hline & Diarreia & 1 & $\mathrm{C} / \mathrm{F}$ & Infusão/Fervimento \\
\hline & Doenças venéreas & 1 & $\mathrm{C} / \mathrm{F}$ & Infusão/Fervimento \\
\hline & Estômago & 1 & $\mathrm{~F} / \mathrm{Ft} / \mathrm{C}$ & Fervimento \\
\hline & Fígado & 1 & $\mathrm{C}$ & Fervimento \\
\hline & Fraqueza & 1 & $\mathrm{C} / \mathrm{F} / \mathrm{Ft} / \mathrm{Fl} / \mathrm{S}$ & Infusão \\
\hline & Impotência sexual & 1 & $\mathrm{C} / \mathrm{F} / \mathrm{Ft} / \mathrm{Fl} / \mathrm{S}$ & Infusão \\
\hline & Infecção da bexiga & 1 & $\mathrm{~F} / \mathrm{Ft} / \mathrm{C}$ & Fervimento \\
\hline & Laxante & 1 & $\mathrm{C}$ & Infusão \\
\hline & Leucemia & 1 & $\mathrm{C} / \mathrm{F} / \mathrm{Ft} / \mathrm{Fl} / \mathrm{S}$ & Infusão \\
\hline & Ovário & 1 & $\mathrm{C} / \mathrm{F}$ & Infusão/Fervimento \\
\hline & Pé de atleta & 1 & $\mathrm{C} / \mathrm{F} / \mathrm{Ft}$ & Fervimento/Tintura \\
\hline & Prisão de ventre & 1 & $\mathrm{C} / \mathrm{F}$ & Infusão/Fervimento \\
\hline & Repor cartilagem & 1 & $\mathrm{~F} / \mathrm{Ft} / \mathrm{C}$ & Infusão \\
\hline & Tuberculose & 1 & $\mathrm{C} / \mathrm{F}$ & Infusão \\
\hline Tecnologia & Bote & 2 & $\mathrm{Cl}$ & - \\
\hline
\end{tabular}

As maiores concordâncias de uso entre os entrevistados foram para o tratamento da gripe $(\mathrm{CUPc}=68)$ e anemia $(C U P c=56)$, seguido pela produção de farinha a partir das sementes do jatobá (CUPc=48) (Tabela 2). Estes resultados concordam com demais estudos que relatam que $H$. courbaril é indicado para o tratamento de gripe (Ferreira et al. 2015) e anemia (Bezerra et al. 2013; Macêdo et al. 2015). A farinha produzida a partir das sementes pode ser consumida naturalmente por apresentar elevado teor de fibra alimentar e grandes quantidades de cálcio e magnésio (Barroso 1991; Shanley e Medina 2005; Batista et al. 2011), desta forma, sugere-se o cultivo do jatobá para o uso familiar, bem como, a sua inclusão na lista de plantas medicinais de interesse do Ministério da Saúde do Brasil que, é composta por 71 espécies de plantas medicinais (Brasil 2009). 
Tabela 2. Concordância quanto ao uso do jatobá (Hymenaea courbaril) na região norte do estado de Mato Grosso.

\begin{tabular}{ccccccc}
\hline Categoria & Uso principal & NICUC & NICUP & CUP & FC & CUPc \\
\hline \multirow{2}{*}{ Medicinal } & Gripe & 25 & 17 & 68,0 & 1,00 & 68 \\
& Anemia & 25 & 14 & 56,0 & 1,00 & 56 \\
\hline Alimento & Farinha do fruto & 12 & 12 & 100 & 0,48 & 48 \\
\hline Madeireira & Madeira construção & 7 & 7 & 100 & 0,28 & 28 \\
\hline & Bronquite & 25 & 4 & 16,0 & 1,00 & 16 \\
& Infecção pulmonar & 25 & 8 & 32,0 & 1,00 & 32 \\
& Infecção geral & 25 & 4 & 16,0 & 1,00 & 16 \\
Medicinal & Reumatismo & 25 & 4 & 16,0 & 1,00 & 16 \\
& Fortalecero pulmão & 25 & 3 & 12,0 & 1,00 & 12 \\
& Imunidade baixa & 25 & 3 & 12,0 & 1,00 & 12 \\
& Infecção urinária & 25 & 3 & 12,0 & 1,00 & 12 \\
& Próstata & 25 & 3 & 12,0 & 1,00 & 12 \\
& Vermífugo & 25 & 3 & 12,0 & 1,00 & 12 \\
\hline
\end{tabular}

NICUC: Número de entrevistados que citou uso da categoria; NICUP: Número de entrevistados citando usos principais; CUP: Índice de concordância de uso principal; FC: Fator de correção; CUPc: CUP corrigida.

\section{Conclusão}

Na região norte do estado de Mato Grosso, embora o jatobá também seja utilizado na alimentação na forma de farinha do fruto, a principal categoria de uso da espécie é a medicinal, utilizando distintas estruturas da planta, principalmente para o tratamento de gripe e anemia, evidenciando, então, que os entrevistados possuem amplo conhecimento sobre a espécie nativa da Amazônia Brasileira.

\section{Agradecimentos}

O presente trabalho foi realizado com apoio da Coordenação de Aperfeiçoamento de Pessoal de Nível Superior - Brasil (CAPES) - Código de Financiamento 001.

\section{REFERÊNCIAS}

Albuquerque UP, Andrade LHC. 2002. Conhecimento botânico tradicional e conservação em uma área de caatinga no Estado de Pernambuco, Nordeste do Brasil. Acta Botanica Brasilica, 16(3):273-285. DOI: 10.1590/S0102-33062002000300004

Albuquerque UP, Lucena RFP. 2004. Seleção e escolha dos informantes. In: Métodos e técnicas na pesquisa etnobotânica. Recife: Livro Rápido/NUPEEA, p. 19-35.

Alvares CA, Stape JL, Sentelhas PC, Gonçalves JLM, Sparovek G. 2013. Koppen's climate classification map for Brazil. Meteorologische Zeitschrift, 22(6):711-728. DOI: 10.1127/0941-2948/2013/0507 
Alvino PO, Silva MFF, Rayol BP. 2005. Potencial de uso das espécies arbóreas de uma floresta secundária, na zona Bragantina, Pará, Brasil. Acta amazônica, 35(4):413-420. DOI: 10.1590/S0044-59672005000400005

Amorozo MCM, Gély A. 1988. Uso de plantas medicinais por caboclos do Baixo Amazonas. Barcarena, PA, Brasil. Boletim do Museu Emílio Goeldi, 4:47-129.

Andrade JKB, Andrade ABA, Azevêdo SMA, Pessoa RMS, Costa Júnior DS. 2013. Levantamento etnobotânico de plantas medicinais no município de Poço de José de Moura-PB. Revista Verde de Agroecologia e Desenvolvimento Sustentável, 8(4):253-257. DOI: $10.18378 /$ rvads.v8i4.2712

APW. Angiosperm phylogeny website. Disponível em: http:/ /www.mobot.org/MOBOT/ research/APweb/. Acesso em: 12 de julho de 2019.

Barroso GM. 1991. Sistemática de angiospermas do Brasil. Viçosa: Imprensa Universitária, 326 p.

Batista AG, Esteves EA, Dessimoni-Pinto NAV, Oliveira LG, Pires ST, Santana RC. 2011. Chemical composition of jatobádo-cerrado (Hymenaea stigonocarpa Mart.) flour and its effect on growth of rats*. Alimentos e Nutrição, 22(2):173-180.

Bezerra GP, Góis RWS, Brito TS, Lima FJB, Bandeira MAM, Romero NR, Magalhães PJC, Santiago GMP. 2013. Phytochemical study guided by the myorelaxant activity of the crude extract, fractions and constituent from stem bark of Hymenaea courbaril L. Journal of Ethnopharmacology, 149(1):62-69. DOI: 10.1016/j.jep.2013.05.052

BFG - The Brazil Flora Group. 2018. Brazilian Flora 2020: Innovation and collaboration to meet Target 1 of the Global Strategy for Plant Conservation (GSPC). Rodriguésia, 69(4): 1513-1527. DOI: 10.1590/2175-7860201869402

Brasil. Ministério da Saúde. Portal da Saúde. 2009. Relação Nacional de Plantas Medicinais de Interesse ao SUS: RENISUS. Disponível em: <http://portalarquivos2.saude.gov.br/images/pdf/2017/junho/06/renisus.pdf>. Acesso em 14 jan 2019.

Campos LZ, Albuquerque UP, Peroni N, Araújo EL. 2015. Do socioeconomic characteristics explain the knowledge and use of native food plants in semiarid environments in Northeastern Brazil?. Journal of Arid Environments, 115: 53-61. DOI: 10.1016/j.jaridenv.2015.01.002

Castellen MS. Avaliação do estado de conservação de populações naturais de Jatobá (Hymenaea courbaril. L) por meio de análise de estrutura genética e correlação espacial. 2005. 104 f. Tese (Doutorado em Ecologia) - Escolar Superior de Agricultura “Luiz de Queiroz”, Universidade de São Paulo, Piracicaba, 2005.

Cunha SA, Bortolotto IM. 2011. Etnobotânica de Plantas Medicinais no Assentamento Monjolinho, município de Anastácio, Mato Grosso do Sul, Brasil. Acta Botanica Brasilica, 25(3):685-698. DOI: 10.1590/S0102-33062011000300022

Fagundes NCA, Oliveira GL, Souza BGD. 2017. Etnobotânica de plantas medicinais utilizadas no distrito de Vista Alegre, Claro dos Poções-Minas Gerais. Fitos, 11(1):1-118. DOI: 10.5935/2446-4775.20170007

Ferreira ALS, Batista CAS, Pasa MC. 2015. Levantamento etnobotânico nas diferentes realidades de ensino. Biodiversidade, 14(3):60-73.

Ferreira FMC, Lourenço FJDC, Baliza DP. 2014. Levantamento etnobotânico de plantas medicinais na comunidade quilombola Carreiros, Mercês-Minas Gerais. Revista Verde de Agroecologia e Desenvolvimento Sustentável, 9(3):205212. 
Fidalgo O, Bononi VLR. 1989. Técnicas de coleta, preservação e herborização do material botânico. São Paulo: Instituto de Botânica, 61 p.

Gandolfo ES, Hanazaki N. 2011. Etnobotânica e urbanização: conhecimento e utilização de plantas de restinga pela comunidade nativa do distrito do Campeche (Florianópolis, SC). Acta Botanica Brasilica, 25(1):168-177. DOI: 10.1590/ S0102-33062011000100020

Guarim Neto G. 1997. A importância da flora amazônica para uso medicinal. Horticultura Brasileira, 15:159-161.

IBGE. Instituto Brasileiro de Geografia e Estatística. Panorama - Alta Floresta, IBGE 2018. Disponível em: < https:// cidades.ibge.gov.br/brasil/mt/alta-floresta/panorama>. Acesso em 08 jan 2019.

Lacerda AEB, Sebbenn AM, Kanashiro M. 2008. Long-pollen movement and deviation of random mating in a low density continuous population of Hymenaea courbaril in the Brazilian Amazon. Biotropica, 40(4):462-470.

Lee YT, Langenheim JH. 1975. Systematics of the genus Hymenaea L. (Leguminosae; Caesalpinioideae; Detarieae). University of California Publications in Botany, 69:1-105.

Lewis GP, Schrire BD, Mackinder BA, Lock JM. 2005. Leguminosae of the World. Royal Botanic Gardens, Kew, 577 p.

Lima HC, Pinto RB. 2015. Hymenaea in: Lista de Espécies da Flora do Brasil. Jardim Botânico do Rio de Janeiro. Disponivel em: <http://floradobrasil.jbrj.gov.br/jabot/floradobrasil/FB22972>. Acesso em 09 Jul 2019.

Lisboa PLB, Terezo EFM, Silva JCA. 1991. Madeiras amazônicas: considerações sobre exploração, extinção de espécies e conservação. Boletim do Museu Paraense Emílio Goeldi, 7(2):521-542.

Macêdo DG, Ribeiro DA, Coutinho HD, Menezes IR, Souza MM. 2015. Práticas terapêuticas tradicionais: uso e conhecimento de plantas do cerrado no estado de Pernambuco (Nordeste do Brasil). Boletín Latinoamericano y del Caribe de Plantas Medicinales y Aromáticas, 14(6):491-508.

Messias MCTB, Menegatto MFM, Prado ACC, Santos BR, Guimarães MFM. 2015. Uso popular de plantas medicinais e perfil socioeconômico dos usuários: um estudo em área urbana em Ouro Preto, MG, Brasil. Revista Brasileira de Plantas Medicinais, 17(1):76-104. DOI: 10.1590/1983-084X/12_139

Moraes Rego CAR, Rocha AE, Oliveira CA, Pacheco FPF. 2016. Levantamento etnobotânico em comunidade tradicional do assentamento Pedra Suada, do município de Cachoeira Grande, Maranhão, Brasil. Acta Agronómica, 65(3):2016. DOI: 10.15446/acag.v65n3.50240

Pasa MC, Soares JJ, Guarim Neto G. 2005. Estudo etnobotânico na comunidade de Conceição-Açu (alto da bacia do rio Aricá Açu, MT, Brasil. Acta Botanica Brasilica, 19(2):195-207. DOI: 10.1590/S0102-33062005000200001

Pereira ZV, Fernandes SSL, Sangalli A, Mussury RM. 2012. Usos múltiplos de espécies nativas do bioma Cerrado no Assentamento Lagoa Grande, Dourados, Mato Grosso do Sul. Revista Brasileira de Agroecologia,7(2):126-136.

Perin, CL. Escola, colonização e formação da identidade do colono: História e memórias da terra prometida de Alta Floresta-MT (1976-1982). 2015. 108f. Dissertação (Mestrado em Educação na Área História da Educação e Memória) - Universidade Federal de Mato Grosso. Cuiabá, 2015. 
Prado ACC, Rangel EB, Sousa HC, Messias MCTB. 2019. Etnobotânica como subsídio à gestão socioambiental de uma unidade de conservação de uso sustentável. Rodriguésia, 70:1-10. DOI: 10.1590/2175-7860201970019

Ramires M, Clauzet M, Begossi A. 2012. Folk taxonomy of fishes of artisanal fishermen of Ilhabela (São Paulo/Brazil). Biota Neotropica, 12(4):29-40. DOI: 10.1590/S1676-06032012000400002

Roboredo D, Bergamasco SMPP, Tosetti LL, Gervásio W, Souza ALA, Domingues TR. (2017). Conflitos socioambientais para a preservação de recursos hídricos na Microbacia Hidrográfica Mariana da Amazônia Matogrossense, 2011 e 2012. Informações Econômicas, 47(3):27-39.

Salman AKD, López GFZ, Bentes-Gama MM, Andrade CMS. 2008. Espécies arbóreas nativas da Amazônia Ocidental Brasileira com potencial para arborização de pastagens. Porto Velho: Embrapa Rondônia. 2008. 20 p. (Documentos, 127).

Seluchinesk RDR. De heróis a vilões: imagem e auto-imagem dos colonos da Amazônia Mato-Grossense. 2008. 263 f. Tese (Doutorado em Desenvolvimento Sustentável) - Centro de Desenvolvimento Sustentável da Universidade de Brasília. Brasília, 2008.

Shanley P, Medina G. 2005. Frutíferas e Plantas Úteis na Vida Amazônica. Belém: CIFOR, Imazon, 300 p.

Silva CG, Marinho MGV, Lucena MFA, Costa JGM. 2015. Levantamento etnobotânico de plantas medicinais em área de Caatinga na comunidade do Sítio Nazaré, município de Milagres, Ceará, Brasil. Revista Brasileira de Plantas Medicinais, 17(1):133-142. DOI: 10.1590/1983 084X/12_055

Silva LL. Substratos para a produção de mudas de jatobá (Hymenaea courbaril L.). 2017. 73f. Dissertação (Mestrado em Ciência e Tecnologia de Sementes) - Faculdade de Agronomia Eliseu Maciel - Universidade Federal de Pelotas. Pelotas, 2017.

Silva MR, Lamarca EV. 2018. Registros etnobotânicos e potenciais medicinais e econômicos do jatobá (Hymenaea courbari). Revista da Universidade Ibirapuera, 15(1):8-12.

Toledo RM. Modelagem espacial do fluxo gênico de sementes de Jatobá (Hymenaea courbari), através de marcadores moleculares, na paisagem fragmentada do Pontal do Paranapanema, SP. 2005. 73f. Dissertação (Mestrado em Ecologia de Agroecossistemas) - Escola Superior de Agricultura "Luiz de Queiroz" - Universidade de São Paulo. São Paulo, 2005.

Vásquez SPF, Mendonça MS, Noda SN. 2014. Etnobotânica de plantas medicinais em comunidades ribeirinhas do Município de Manacapuru, Amazonas, Brasil. Acta Amazonica, 44(4):457-472. DOI: 10.1590/1809-4392201400423 